\title{
Pasteurela Endocarditis with Spine Involvement - A Case Report and Review of Literature
}

Ofir Elalouf ${ }^{1}$, Yaakov Varkel', Rima Feldman ${ }^{1}$, Danny Glikman ${ }^{1}$ and Zvi H Perry ${ }^{2,3 *}$

${ }^{1}$ West Galilee Naharia Hospital, Internal Ward C, Israel

${ }^{2}$ Department of Epidemiology and Health Services Evaluation, Ben-Gurion University of the Negev, Beer-Sheva, Israel and Surgical ward A, Soroka Medical Center, Beer Sheva, Israel

${ }^{3}$ Surgery ward A, Soroka Medical Center, Israel

\begin{abstract}
Pasteurella multocida is the causative agent of numerous economically relevant diseases worldwide. Pasteurella multocida has been known to cause infections in humans, the most familiar being soft tissue infection after animal bites. Systemic infections are rare and endocarditis is exceptionally rare.

We report about a 72 year old woman with prosthetic biologic aortic valve was admitted to West Galilee Naharia Hospital because of 10 days long fever after a domestic cat scratch in her left palm. During her stay the patient continued to have spiking fever accompanied by shivering despite the antibiotic therapy, she had worsening dyspnea, tachypnea, feet edema and cough. Due to the findings listed above another trans thorathic echocardiography was performed on the $11^{\text {th }}$ day of her hospitalization, which showed endocarditis of the prosthetic aortic. On the $42^{\text {nd }}$ day an MRI exam was done that showed an inflammatory process that involved the I5-s1 vertebras and the intervertebral disc. We believe our report is the second documented case of Pasteurella multocida endocarditis that was complicated by osteomyelitis, and we contemplate upon this rare occurrence and its implications.
\end{abstract}

Keywords: Endocarditis; Pasteurela multocida; Risk factors; Cats

\section{Case Report}

A 72 year old woman with prosthetic biologic aortic valve was admitted to West Galilee Naharia Hospital because of 10 days long fever after a domestic cat scratch in her left palm.

Approximately for 1 year earlier she went through an aortocoronary bypass and a biological aortic valve replacement surgery due to ischemic heart disease and senile aortic stenosis. She underwent the surgery without major complications, and returned to fully active daily life.

10 days before admission the patient was scratched by a domestic cat in her left palm. After the scratch there was no swelling, local redness, or any other local reaction. One day later macular linear lines developed all along the left arm which disappeared a few days later. Fever up to $39.5^{\circ} \mathrm{C}$ degrees developed simultaneously with the rash and was accompanied by shivering and chills. She was treated ambulatory by Ampicilin-calvulonate for 5 days with no improvement, and was referred to our hospital for further evaluation.

The patient had a history of diabetes mellitus type 2 with diabetic retinopathy and was treated with insulin injections. In addition, the patient suffered from arterial hypertension and hypercholesterolemia that were balanced with pharmacotherapy. She had primary hypothyroidism that was balanced with thyroxin.

The patient was a new immigrant to Israel from Russia and lived in Acre. She was married and had no children. The patient's family owned a cat that was vaccinated properly. She had not travelled outside the country and had no contact with ill persons. She didn't use tobacco or alcohol in the past, and didn't have any drug allergy. Medications taken on daily basis included thyroxin, furosemide, aspirin, atenolol, clonidine, amlodipine, losartan, simvastatin and SC insulin.

On examination, the temperature was $37.0^{\circ} \mathrm{C}$, pulse 82 beats per minute, and the blood pressure $140 / 90 \mathrm{mmHg}$; the respirations were 15 per minute. The oxygen saturation was $92 \%$ while breathing ambient air. She appeared well but mildly pale, with no tachypnea. She was obese with a BMI of $37 \mathrm{~kg} / \mathrm{m}^{2}$. There was no laryngeal erythema or tonsilar exudates. No cervical or axillary lymphadenopaty was noted. A well healed sternotomy scar was noted. Auscultation of the chest revealed normal breath sounds. There were regular heart sounds with $3 / 6$ grade systolic murmur heard best on left sternal border, and which radiated to the neck. The jugular venous pressure was normal. There were no Osler's nodes, Janeway's lesions, splinter hemorrhages or peripheral edema. At this time the rash in the left arm was disappeared. Eye fundoscopy was normal. Specimens of blood were sent for culture. ECG showed sinus rhythm with no acute signs of ischemia. Radiograph of the chest was normal.

In the next morning, a temperature of $40^{\circ} \mathrm{C}$ was measured. A Trans thorathic echocardiography was done, which didn't demonstrate any vegetations. A few hours later a growth of gram negative rods bacteria in four different blood culture bottles was noted. Treatment with Imipenem was initiated immediately.

On the $3^{\text {rd }}$ hospitalization day, the patient started to complain of diffuse low back pain. A computed tomography of the chest and the abdomen didn't show any abscess or other findings that could explain the prolonged fever. Trans esophageal echocardiography was performed on the same day and a mobile $0.9 \mathrm{~cm}$ vegetation of the posterior leaflet connected to the calcified mitral valve was found, with an aortic gradient of $45 \mathrm{mmHg}$. Final identification of the blood cultures demonstrated growth of Pasteurella multocida bacteria. After confirmation that the bacterium was susceptible for penicillin, the

*Corresponding author: Zvi Perry, MD, The Moshe Prywes Center for Medical Education, Ben-Gurion University of the Negev, PO Box 653, Beer-Sheva 84105 Israel, Tel: +972-8-647-7415; Fax: +972-8-647-7633; E-mail: zperry@bgu.ac.il

Received November 28, 2012; Accepted December 28, 2012; Published December 31, 2012

Citation: Elalouf O, Varkel Y, Feldman R, Glikman D, Perry ZH (2013) Pasteurela Endocarditis with Spine Involvement-A Case Report and Review of Literature. J Clin Case Rep 3:239. doi:10.4172/2165-7920.1000239

Copyright: $\odot 2013$ Elalouf $\mathrm{O}$, et al. This is an open-access article distributed under the terms of the Creative Commons Attribution License, which permits unrestricted use, distribution, and reproduction in any medium, provided the original author and source are credited. 
Imipenem treatment was stopped and a therapy with maximal dose of intravenous penicillin was initiated.

On the $4^{\text {th }}$ hospitalization day the patient had a bone scintigraphy for her back pain which was negative for osteomyelitis. Concurrently a revision of the computed tomography with a special focus on the spine was made. The patient was evaluated by our spine surgeon that concluded that the pain is probably due to degenerative changes, and analgesic therapy was recommended.

During the next few days the patient continued to have spiking fever accompanied by shivering despite the antibiotic therapy, she had worsening dyspnea, tachypnea, feet edema and cough. Due to the findings listed above another trans thorathic echocardiography was performed on the $11^{\text {th }}$ day of her hospitalization. The test didn't demonstrate any vegetation but showed a significant magnification of the aortic gradient ( $40 \mathrm{mmHg}$ vs. $104 \mathrm{mmHg}$ ). The increment in the gradient was considered to be attributed to the endocarditis of the prosthetic aortic valve and a surgical procedure was suggested but was rejected by the patient. On the $13^{\text {th }}$ day another Trans esophageal echocardiography was performed that demonstrated two small (0.6$0.7 \mathrm{~cm}$ ) vegetation on the anterior leaflet of the mitral valve, but no vegetations were seen on the aortic prosthetic valve.

The patient continued to complain of worsening back pain despite aggressive opiate therapy, the pain prevented her from getting up of bed. It is important to note that on the $5^{\text {th }}$ day the patient developed urinary retention and a urinary catheter was placed and therapy with alpha blocker was initiated. A reevaluation by a spine sergeant was performed and there were no new recommendations regarding further evaluation or therapy.

From the $12^{\text {th }}$ day of admission until the $40^{\text {th }}$ day, the patient continued to have sub febrile fever with a spiking fever up to 38.3 once every few days, repeated blood cultures were negative. Clinically her hemodynamic and respiratory situation gradually improved, she didn't complain of dyspnea, and her feet edema reduced. She continued to complain about her back pain and still was confined to bed.

On day 36 a repeated bone scintigraphy with Tc-MDP was performed and demonstrated an increased comprehension of S1 in comparison to the first exam. A repeated computed tomography of the thoracic-lumbar spine and the pelvis demonstrated bony destruction of the inferior aspect of L5 and the superior aspect of S1 and fullness of the surrounding soft tissue. On the $42^{\text {nd }}$ day an MRI exam was done that showed an inflammatory process that involved the 15 -s1 vertebras and the intervertebral disc. The imaging results correlated primarily to osteomyelitis of the vertebras and spondylodiscitis. An infectious disease expert recommended switching the antibiotic therapy to an antibiotic with a better penetrability to bone, and an intra venous therapy with ceftriaxone was initiated. The patient continued to improve and 2 weeks after, she got out of bed and started to walk. Her fever subsided and on her discharge, she returned to her basic ADL function.

\section{Discussion}

Pasteurella (P) multocida is the causative agent of numerous economically relevant diseases worldwide [1]. Pasteurella is a Gramnegative coccobacillus found in $70-90 \%$ of oral cavities of cats, and as well, is isolated from the digestive systems of dogs, rats, rabbits, monkeys, and other animals. In humans P. multocida is of increasing importance, causing wound infections, and even septicemia, meningitis, and endocarditis [1]. It is worth mentioning that human infection with Pasteurella multocida is the leading cause of animal bite wound infection [2]. Pasteurella multocida has been known to cause infections in humans, the most familiar being soft tissue infection after animal bites. Systemic infections are rare and endocarditis is exceptionally rare. Infective endocarditis (IE) due to Gram-negative bacteria is a rare occurrence by itself, with a relative frequency of less than $10 \%$ compared to that caused by Gram-positive bacteria [3]. Research has shown that Gram-negative endocarditis presents as an acute form in $64 \%$ of cases and affects the aortic as often as the mitral valves. Contact with an animal is documented in $65 \%$ of cases. Pasteurella multocida is the most frequent species in this infection. The total death rate is $40 \%$ and can reach $57 \%$ of cases in case of immunedepression. In a retrospective study of Pasteurella species bacteremia (PSB) episodes, Pasteurella multocida was the predominant species, identified in $70 \%$ of all isolations. All the patients were adults over 50 years old and all had underlying illnesses causing comorbidity or some degree of immunocompromise, with cardiovascular and hypertensive conditions being the most frequent; only one patient had liver cirrhosis. In all cases, except one, contact or coexistence with dogs or cats was documented.

Literature has seen some case reports upon Pasteurella multocida endocarditis, as early as the 1970's, when Lehmann et al. [4], described a 51-year-old previously healthy man developed who endocarditis with low grade fever, Aortic insufficiently and multiple arterial emboli. An atypical strain of Pasteurella multocida was isolated from blood cultures, and the researchers warned that $P$. multocida should be regarded as one of the possible causes of subacute bacterial endocarditis. From that time onwards a growing amount of evidence has accumulated to show that Pasteurella multocida is a central player in gram negative endocarditis [3,5-10].

To our best of knowledge this is the second documented case of Pasteurella multocida endocarditis that was complicated by osteomyelitis. The previous reported case was reported by Sorbello et al. [9] which reported a case of endocarditis due to Pasteurella dagmatis complicated by vertebral osteomyelitis in a 55-year-old former alcoholic woman.

The issue at hand now that we know endocarditis from Pasteurella multocida is plausible, is how are at risk for this dreaded and sometimes fatal disease?

As mentioned, Pasteurella multocida can cause septic arthritis, osteomyelitis, pneumonia, endocarditis, meningitis, and septicemia [11]. Over $90 \%$ of human infections are cases of wound infections or abscesses related to a bite, scratch, or licking of skin lesions by a cat or dog $[5,6]$. Severe systemic diseases such as pneumonia, lung abscess, peritonitis, endocarditis, meningitis and sepsis are also well known, especially in patients with underlying medical conditions [2,5].

Treatment: As mentioned above, emphasis should be placed on preventive measures related to care and hygiene among individuals with pets [12]. Susceptibility and outcome of primary treatment with penicillins and other betalactams shows that they are still appropriate therapy $[6,12]$, but one must remember that protracted treatment with high doses of penicillin $\mathrm{G}$ is necessary to eradicate the infection. Today it is widely adapted but not yet consensual he use of medico-surgical treatment $[7,8,13]$ for the offended cardiac lesions.

The bad prognosis of this infection, justifies an early diagnosis and a rapid treatment. Pasteurella multocida is a normal inhabitant of the oral cavity of dogs and cats. Therefore, people who have frequent contact with these animals should be examined periodically for signs of infection [8]. Drenjancevic et al. [5] emphasize the need for primary 
Citation: Elalouf O, Varkel Y, Feldman R, Glikman D, Perry ZH (2013) Pasteurela Endocarditis with Spine Involvement-A Case Report and Review of Literature. J Clin Case Rep 3:239. doi:10.4172/2165-7920.1000239

healthcare to provide more information to patients at risk of infections from contact with animals and to warn them about the possible consequences of injuries, even when the animals are pets. Hazouard et al. [14] for example, believe that because P. multocida could be fulminant in immunocompromised, they propose for these patients reducing exposure to cats and hand-washing after exposure if wounds are present. In any case, a high index of suspicion and early diagnosis, especially in immunocompromised patients, are warranted because the disease is potentially life threatening, yet is a readily treatable infection [2].

\section{References}

1. Ewers C, Lubke-Becker A, Wieler LH (2004) Pasteurella: insights into the virulence determinants of a heterogenous bacterial type. Berl Munch Tierarztl Wochenschr 117: 367-386

2. Hombal SM, Dincsoy HP (1992) Pasteurella multocida endocarditis. Am J Clin Pathol 98: 565-568.

3. Reinsch N, Plicht B, Lind A, Janosi RA, Buck T, et al. (2008) Recurrent infective endocarditis with uncommon Gram-negative Pasteurella multocida and Pseudomonas aeruginosa: a case report. J Heart Valve Dis 17: 710-713.

4. Lehmann V, Knutsen SB, Ragnhildstveit E, Skagseth E, Solberg CO (1977) Endocarditis caused by Pasteurella multocida. Scand J Infect Dis 9: 247-248.

5. Drenjancevic IH, Ivic D, Drenjancevic D, Ivic J, Pelc B, et al. (2008) Fatal fulminant sepsis due to a cat bite in an immunocompromised patient. Wien Klin Wochenschr 120: 504-506.
6. Naba MR, Araj GF, Kanafani ZA, Kanj SS (2009) First case of Pasteurella multocida endocarditis of the tricuspid valve: a favorable outcome following medical treatment. Int J Infect Dis 13: e267-269.

7. Fayad G, Modine T, Mokhtari S, Legout L, Decoene C, et al. (2003) Pasteurella multocida aortic valve endocarditis: case report and literature review. J Heart Valve Dis 12: 261-263.

8. Fukumoto $Y$, Moriyama Y, Iguro $Y$, Toda R, Taira A (2002) Pasteurella multocida endocarditis: report of a case. Surg Today 32: 513-515.

9. Sorbello AF, O'Donnell J, Kaiser-Smith J, Fitzharris J, Shinkarow J, et al. (1994) Infective endocarditis due to Pasteurella dagmatis: case report and review. Clin Infect Dis 18: 336-338.

10. Thamlikitkul V, Sangruchi T (1990) Pasteurella multocida infective endocarditis: a case report. J Med Assoc Thai 73: 704-706.

11. Per H, Kumandas S, Gumus H, Ozturk MK, Coskun A (2010) Meningitis and Subgaleal, Subdural, epidural Empyema due to Pasteurella Multocida. J Emerg Med 39: 35-38.

12. Felix M, Tallon P, Salavert M, Navarro V, Breton JR, et al. (2003) Bacteremia due to Pasteurella spp.: a rare process in our hospital over the last 8 years. Enferm Infecc Microbiol Clin 21: 334-339.

13. Camou F, Guisset O, Pereyre S, Gabinski C, Viallard JF (2005) Endocarditis due to Pasteurella sp. Two cases. Med Mal Infect 35: 556-559.

14. Hazouard E, Ferrandiere M, Lanotte P, Le Marcis L, Cattier B, et al. (2000) Septic shock caused by Pasteurella multocida in alcoholic patients. Probable contamination of leg ulcers by the saliva of the domestic cats. Presse Med 29 : 1455-1457. 